\title{
Power Control for Wireless Packet Voice With Application to EDGE System
}

\author{
Justin C.-I. Chuang, Kin K. Leung, Xiaoxin Qiu, Shailender Timiri and Li-Chun Wang \\ AT\&T Labs - Research \\ 100 Schulz Drive \\ Red Bank, NJ 07701 \\ \{justin, kkleung, xqiu, lichun\}@ research.att.com \& shailender.timiri@attws.com
}

\begin{abstract}
In this paper, using the EDGE system as an example, we apply a Kalman-filter power control method based on interference tracking and prediction to packet voice service in wireless networks. Our results reveal that power control significantly improves the spectral efficiency by enabling $1 / 3$ frequency reuse while maintaining a stringent requirement of $2 \%$ packet loss probability for voice service. More specifically, for allocated spectrum of 1.8, 3.6 and $5.4 \mathrm{MHz}$, the $1 / 3$ reuse with the Kalman power control can yield $102.5 \%, 49.5 \%$ and $32.5 \%$ improvement in spectral efficiency, respectively, over 3/9 reuse (regardless of whether or not power control is used). We also find that the Kalman method provides $20 \%$ additional spectral efficiency when compared with a traditional SIR power control method, and the former method is more robust than the latter for increased power update period. The protocol requirements for the implementation of the Kalman method in the EDGE system are also discussed.
\end{abstract}

\section{INTRODUCTION}

Currently, the ETSI is in the process of establishing the protocol standards for the Enhanced Data rates for GSM Evolution (EDGE) system [SAE98] as the third generation time-division-multiple-access (TDMA) wireless network. Using packet-switching technology and the existing 200 $\mathrm{kHz}$ GSM channels, the EDGE system employs a linkadaptation technique to support the highest data rate approaching $480 \mathrm{kbps}$. Due to the advantages and flexibility of packet switching, the EDGE system is expected to serve as a platform for integrated services including at least packet voice and data. For voice service, each call alternates between talk-spurt and silence period. To increase network capacity, radio resources are assigned to a call only when it has packets to transmit during talk-spurt periods. This technique is known as statistical multiplexing.

Dynamic transmission power control has been widely studied and practiced to combat and manage interference in cellular radio networks; see [Z92], [RZ98] and [UY98]. Particularly for time-division-multiple-access (TDMA) wireless networks like the EDGE system, power control has been shown to be useful in improving network performance and capacity. Power control is also essential in codedivision-multiple-access (CDMA) networks; see [AC93] and [RZ98]. Existing power control algorithms can be classified as signal-based and signal-to-interference-ratio (SIR) based power control. Signal-based control [W93] [HWJ97] adjusts transmission power based on the received signal strength, while the SIR-based power control [Z92] [FM93] changes power according to the ratio of signal and co-channel interference (possibly plus noise) power levels. It is known that SIR-based power control yields higher performance gain than the signal-based control, although the former is more complicated in implementation due to its required frequent exchange of control information between a receiver to its transmitter.

Mathematically, SIR-based power control can be represented as an iterative algorithm that repeatedly adjusts transmission power according to previous SIR measurements. Due to the nature of iterations, SIR-based algorithms typically perform well for calls with relatively long holding time. Recently, for application in wireless packet networks with bursty transmission, [L99] proposes a power-control method that is based on measurements and prediction of interference power by use of a Kalman filter. Results in [L99] reveal the potential performance gain of power control by tracking of interference power.

The purposes of this work are twofold. First, we explore the applicability and quantify the performance gain of the Kalman power control for packet voice service in the EDGE system. Second, we present at a high level the exchange of control information needed for the power-control method. In addition, we also examine the impacts of the Kalman method on the EDGE protocol design. We should emphasize that although the EDGE system is used as an example to make our discussion concrete, results and ideas in this paper are generally applicable to other packet wireless networks.

The organization of the rest of this paper is as follows. We outline in Section II the Kalman power control and its pertinent operations of the EDGE system. In Section III, we use computer simulation to quantify the performance gain of the Kalman power control for packet voice service in terms of spectral efficiency and coverage. We also compare performance between the Kalman and SIR-based power control. Implementation considerations of the Kalman 
power control on the EDGE system are discussed in Section IV. Finally, we present our conclusion and future work in Section V.

\section{EDGE System AND PoWer ConTrol}

The EDGE system makes use of existing $200 \mathrm{KHz}$ channels (carriers) in the GSM. Each carrier is divided into time slots and 8 adjacent slots form a TDMA frame, which lasts for $4.615 \mathrm{msec}$ [R96]. In the current proposal, the EDGE system has nine modulation and coding schemes (MSC's) [E99a], and uses a link-adaptation technique to adapt packet transmission to one of the schemes according to the link quality. Since we focus on the packet voice in the EDGE system here, it is assumed that packets of all calls are transmitted at MSC-2 (using GMSK modulation) to achieve robustness and a data rate of $11.2 \mathrm{Kbits} / \mathrm{sec}$ per time slot, adequate for voice applications. Furthermore, this paper considers only voice service in the system and the use of power control for integrated voice and data services will be studied in our subsequent work.

Typically, vocoder generates one voice packet (also known as voice frame) per $20 \mathrm{msec}$. Each voice packet is treated as a radio-link-controller (RLC) block. In turn, each RLC block is divided into four bursts, which are transmitted in a designated time slot over four successive TDMA frames, one burst per frame. For simplicity, we treat these four time slots carrrying the four bursts of a voice packet as a single time slot in the rest of this paper.

For packet voice service, each call alternates between talking mode, at which voice packets are generated periodically by the vocoder, and silence period for both downlink and uplink transmission. When a mobile station (MS) starts a talk spurt for uplink transmission, it first sends a signaling message to its base station (BS) to request a quick assignment of a voice channel (i.e., a time slot on a particular frequency carrier) to carry the newly generated voice packets. The BS chooses one available channel for the request, and instructs via a downlink control channel the MS to start transmission in that time slot. At the end of the talk spurt, the latter channel is relinquished for use by other calls. Similarly, when a talk spurt is started for downlink transmission, the BS sends a paging message over a control channel to the MS, and instructs the latter to receive its packets on a particular voice channel. Upon receiving an acknowledgment from the MS via a control channel, the BS starts packet transmission in the chosen channel. Again, the channel is released upon the completion of the talk spurt.

It is worth noting that the response time of existing GSM protocols are too long for supporting such fast resource assignment on a per talk-spurt basis. For this reason, [QCC00] recently proposes a set of new signaling protocols to maintain satisfactory voice clipping (i.e., loss of the first few packets of a talk spurt). Since the focus of this study is on power control, we assume that the new protocols are available such that voice clipping is not an issue and our primary performance concern is the overall loss probability of voice packets for each call.

The Kalman-filter method [L99] is used to control transmission power. For each MS with power on, it continuously measures the interference-plus-noise power (to be referred to as interference power for brevity) for a small set of voice channels, which is being used or may be used to carry future voice traffic from the BS to the MS. These measurements are continuously fed into a Kalman filter to predict future interference power on these channels.

Since voice packets of a talk spurt associated with each call are transmitted in the same time slot over successive TDMA frames (i.e., a voice channel) in the EDGE system, let us focus only on that time slot and index the TDMA frames by $n$. For a transmitter, either a MS or BS, its transmission power in the time slot of frame $n$ is set to be

$$
p(n)=\min \left\{\gamma * \tilde{I}(n) / g(n), p_{\max }\right\}
$$

Where $\gamma^{*}$ is the SINR target for the voice service using the MSC-2, $\tilde{I}(n)$ is the interference power (in $\mathrm{mW}$ ) for the slot in frame $n$ predicted by the Kalman filter, $g(n)$ is the path gain between the transmitter and the intended receiver in frame $n$, and $p_{\text {max }}$ is maximum power level. Readers are referred to [L99] for the details of the Kalman method. By use of a control channel associated with each call (particularly for handoff purposes), the path gain $g(n)$ can be estimated and known to both the MS and its BS in the EDGE system.

Actually, the Kalman method represents a closed-loop control that requires exchange of control information between the receiver and the transmitter. Without getting into the protocol details at this point, such exchange of information can be made possible by including the pertinent information in appropriate control messages. A possible scenario of message exchanges is outlined in Figures 1 and 2. (Additional discussion on the control messages is presented in Section IV.)

As shown in Figure 1, when an established call is in a silent period for downlink transmission, the associated MS continuously measures and predicts by use of the Kalman filter the interference power on several channels, which may be used to transmit the next talk spurt on the downlink. When the next talk spurt starts, the BS sends a paging message to the MS over a control channel. In turn, the MS includes the predicted interference power for a few voice channels in the paging response message. The BS selects (possibly making use of the interference predictions) and informs the MS of the chosen channel in the resourceassignment message. Then, the BS can start transmitting voice packets. While receiving packets, the MS continues to 
measure and predict interference power for the given set of channels, including the channel where packets are received. Periodically, it sends the interference prediction for the receiving channel back to the $\mathrm{BS}$ via a control channel. With the new prediction $\tilde{I}(n)$, the BS adjusts its transmission power according to (1). Similar operations apply to the uplink transmission as shown in Figure 2.

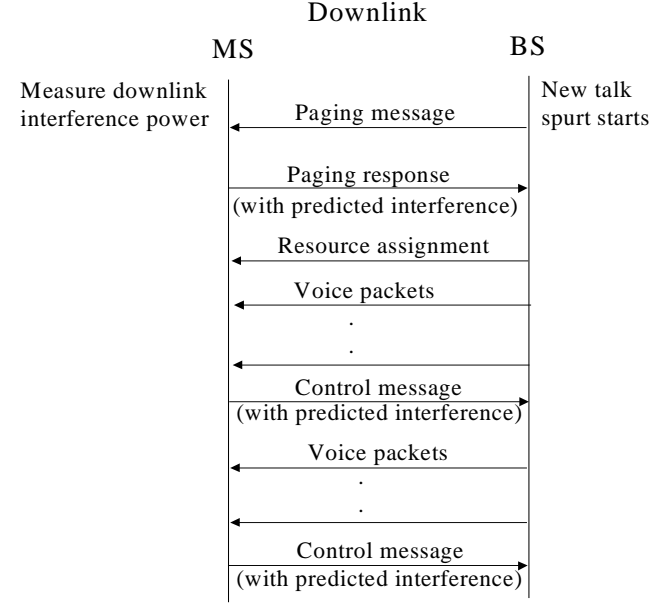

Figure 1. Control Messages for Downlink Transmission

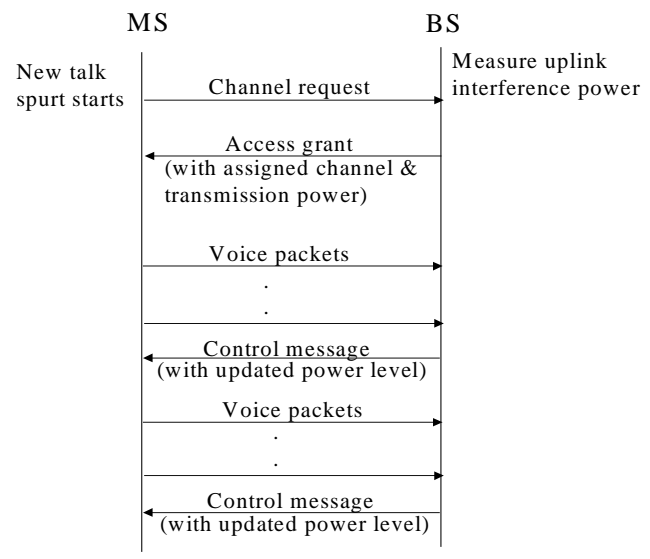

Figure 2. Control Messages for Uplink Transmission

\section{Performance Study}

\section{A. Simulation Model}

We use computer simulation to quantify the performance of Kalman power control for packet voice service. A total of 37 cells in a traditional hexagonal layout is simulated. Each cell is divided into three sectors, each of which is served by a BS antenna at the center of the cell. The beamwidth of each BS antenna is 60 degrees, while MS's have omnidirectional antennas. The BS antenna has a front-to-back gain ratio of $25 \mathrm{~dB}$. Frequency reuse factors of $1 / 3$ and 3/9 are considered in the simulation. Each radio link between a MS and a BS is characterized by a path-loss model with an exponent of 3.5 and lognormal shadow fading with a dB standard deviation of 6 . Cell radius is assumed to be $1 \mathrm{Km}$ and the path loss at $100 \mathrm{~m}$ from the cell center is $-73 \mathrm{~dB}$ and thermal noise at each receiver is fixed and equal to -116 $\mathrm{dBm}$ (for the $200 \mathrm{kHz}$ GSM channel with $5 \mathrm{~dB}$ noise factor). Each sector is populated with $100 \mathrm{MS}$ 's randomly and each of them selects the BS that provides the strongest signal power. Results reported below are aggregated from six repeated runs, each of them lasted for a fraction to one million time slots. All MS's remain at the fixed locations throughout the simulation. For simplicity, we assume that timing for all co-channel sectors are synchronized at the slot boundary for transmission.

The MCS-2 is used for transmitting voice packets. For each packet transmission, the SINR is measured at the receiver. The SINR measurement is rounded to its closest integer value in $\mathrm{dB}$ and the packet error is determined based on the SINR value and the corresponding error probability (which are averaged over Rayleigh fading with cyclic-frequency hopping) in Table 1 [C99]. Packet error probability is zero if the SINR exceeds $23 \mathrm{~dB}$. With these error results, $\gamma *$ for power control in (1) is selected by repeated test runs so that the chosen target minimizes the overall packet error rate (i.e., averaged over all MS's). Using this approach, we found that $\gamma^{*}=15 \mathrm{~dB}$ provides the best results.

\begin{tabular}{|c|c|}
\hline $\begin{array}{l}\text { Measured } \\
\text { SINR }(\mathrm{dB})\end{array}$ & $\begin{array}{c}\text { Packet Error } \\
\text { Probability }\end{array}$ \\
\hline 0 & 0.9001 \\
\hline 1 & 0.8364 \\
\hline 2 & 0.7547 \\
\hline 3 & 0.6610 \\
\hline 4 & 0.5649 \\
\hline 5 & 0.4638 \\
\hline 6 & 0.3645 \\
\hline 7 & 0.2748 \\
\hline 8 & 0.2024 \\
\hline 9 & 0.1410 \\
\hline 10 & 0.0949 \\
\hline 11 & 0.0659 \\
\hline 12 & 0.0452 \\
\hline 13 & 0.0274 \\
\hline 14 & 0.0171 \\
\hline 15 & 0.0103 \\
\hline 16 & 0.0070 \\
\hline 17 & 0.0045 \\
\hline 18 & 0.0026 \\
\hline 19 & 0.0016 \\
\hline 20 & 0.0010 \\
\hline 21 & 0.0006 \\
\hline 22 & 0.0004 \\
\hline 23 & 0.0002 \\
\hline 24 & 0.0000 \\
\hline
\end{tabular}

Table 1. Packet Error Probability Vs. SINR 
The durations of a talk spurt and a silent period for each call are exponentially distributed with an average of 1 and 1.35 sec, respectively. As a packet is generated every $20 \mathrm{msec}$, the number of packets in a talk spurt is geometrically distributed with an average of 50. We assume that mechanisms such as those in [QCC00] for fast channel assignment on a per talk-spurt basis are used to maintain satisfactory voice clipping performance. When a talk spurt starts, the BS randomly assigns one of its available channels to carry the talk spurt. If no channel is available, the entire talk spurt is assumed to be lost (or blocked).

Since each sector typically has tens of voice channels and since each of the MS's in the system needs to measure and track interference power continuously, the simulation model requires a very long CPU time. To make the model efficient, we simulate the interference measurement and tracking for only one voice channel in all co-channel sectors. For example, consider downlink transmission where a talk spurt is sent to a MS in a sector over the channel. Following that, the channel remains idle in the sector for a random duration of time, which is geometrically distributed with a mean matching a given traffic load. After the idle period, the BS starts a transmission of a new talk spurt for another randomly selected MS in the sector. The packet error statistics are collected for each MS over the entire simulation run. We have verified that this simplifying approach essentially yields the same results as if the details of multiple channels and the random channel assignment scheme are simulated.

\section{B. Performance Results}

We define that the quality of packet voice service is satisfactory if a) the blocking probability of both new call and talk spurts due to channel unavailability is less than $2 \%$, and b) packet error rate does not exceed 2\% [FHC99] for calls associated with at least $90 \%$ of MS's in each sector (i.e., a $90 \%$ coverage requirement). By assuming that talk spurts arrive according to a Poisson process, the voice capacity is the maximum traffic load in Erlangs while maintaining the satisfactory service quality.

As a comparison, in addition to the Kalman power control, we also studied the voice performance of the traditional SIR power control [FM93]. Specifically, the transmission power of the first packet of a talk spurt is chosen to fully compensate its path loss and shadow fading. Power for the subsequent packets (indexed by $n$ ) are adjusted according to

$$
p(n)=\min \left\{p(n-1) \gamma^{*} / \beta(n-1), p_{\max }\right\}
$$

Where $\beta(n-1)$ and $\gamma^{*}$ are the measured SINR for packet $n-1$ and the target SINR, respectively. (It is worth noting that an exponential smoothing can be applied to the SINR measurements in the method proposed in [FM93]. Since the measurements are assumed to be error free in this study, the smoothing is not included in (2) to improve the speed of convergence.)

In Figure 3, we consider that the voice service is allocated with 1.8, 3.6 and 5.4 MHz spectrum. Correspondingly, for the $1 / 3$ reuse, each sector is assigned with 24 (i.e., 3 carriers times 8 slots), 48 and 72 voice channels. Similarly, for the $3 / 9$ reuse, each sector has one third of these many channels. Figure 3 shows the downlink spectral efficiency in terms of Erlang/cell/MHz for the voice service with the Kalman method, SIR method and no power control. Results in the figure assume that the SINR and interference power can be measured accurately, and that the measurements for one voice packet can be used to control transmission power for the next packet (i.e., the measurement and control feedback delay is assumed to be less than $20 \mathrm{msec}$ ).

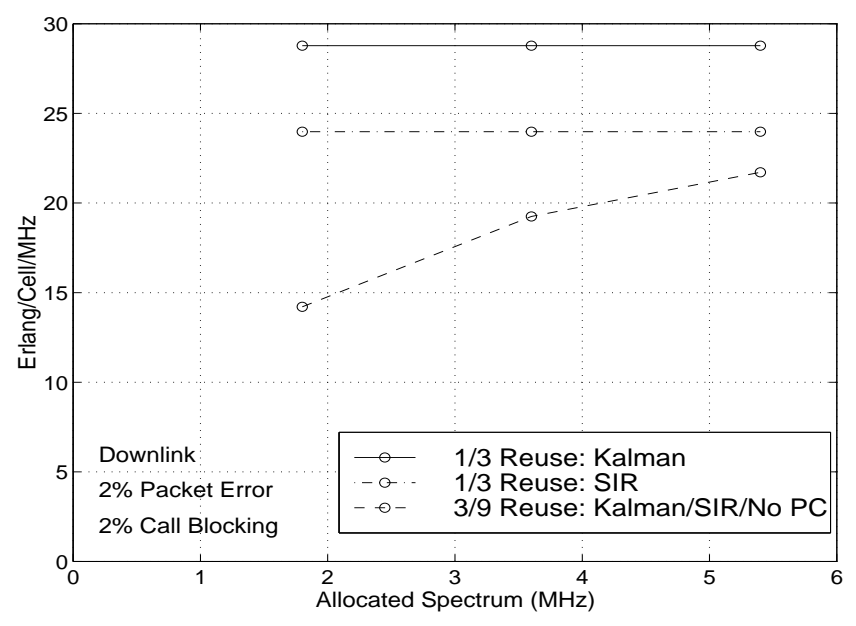

Figure 3. Spectral Efficiency for the 1/3 and 3/9 Reuse

We make several observations from the figure. First, our results for the 3/9 reuse show that the Kalman and SIR power control enable each voice channel to carry $100 \%$ of traffic load, and no power control can support $70 \%$ traffic while meeting the required $2 \%$ packet loss probability for $90 \%$ of MS's in each sector. However, the limiting factor for the spectral efficiency of the 3/9 reuse is the blocking probability for talk spurts. As a result, the Kalman method, SIR method and no power control yield the same spectral efficiency, as shown in Figure 3. As the allocated spectrum increases, the trunking efficiency and thus the spectral efficiency are improved. On the other hand, since the $1 / 3$ reuse is the lowest reuse factor, each sector is allocated with the maximum possible number of channels for a given spectrum allocation, thus avoiding the trunking inefficiency. The limiting factor for the voice capacity in the $1 / 3$ reuse is the packet loss probability, which is mainly determined by the carried traffic load of each channel and thus the interference. In this case, since the voice capacity is almost directly proportional to the maximum feasible load on each 
channel (as traffic load is balanced among all channels by the random channel assignment), the spectral efficiency become independent of the actual spectrum allocation, as shown in Figure 3.

Second, our results reveal that the Kalman and SIR power control support each channel to carry a maximum of $30 \%$ and $25 \%$ of traffic load, respectively, to maintain the stringent $2 \%$ loss probability. For the $1 / 3$ reuse, the spectral efficiency for the power control methods is 28.78 and 23.98 Erlangs/cell/MHz, respectively. That is, the Kalman power control yields about $20 \%$ improvement on spectral efficiency when compared with the SIR control. Furthermore, for the 1.8, 3.6 and $5.4 \mathrm{MHz}$ spectrum allocation, the $1 / 3$ reuse with the Kalman power control provides $102.5 \%, 49.5 \%$ and $32.5 \%$ improvement in spectral efficiency, respectively, over the $3 / 9$ reuse with the Kalman method, SIR method or no power control. We choose to compare with the $3 / 9$ reuse here because it gives a capacity higher than the $1 / 3$ reuse with no power control.

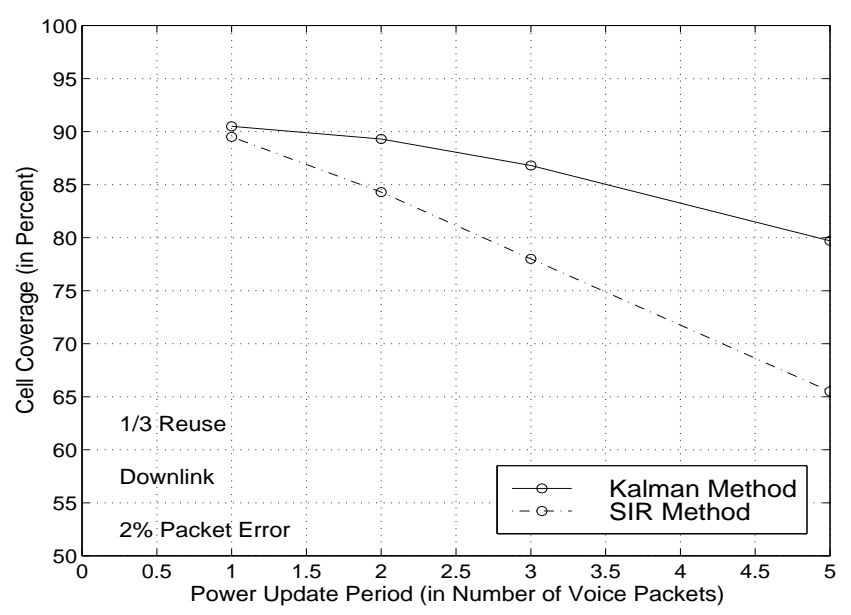

Figure 4. Impact of Power Update Period

Besides the $20 \%$ improvement of the Kalman power control over the SIR control in Figure 3, our results also reveal that the former method is more robust than the latter in terms of coverage. More specifically, Figure 4 shows the impact of coverage due to power update period for both power control methods. To obtain these results, MS's continue to measure and track interference power for each packet transmission, but the transmission power is updated periodically according to the given update period. As depicted in the figure, the $90 \%$ coverage requirement is met by the methods when the system runs at their respective capacity of $30 \%$ and $25 \%$ traffic load. However, at their capacity load, if transmission power is updated every two voice packets, the coverage for the Kalman and SIR method reduces to $89.3 \%$ and $84.3 \%$, respectively. As shown in the figure, additional increase in the power update period further degrades the coverage performance. Nevertheless, these results confirm that the Kalman power control is more robust than the SIR as far as power update period is concerned.

The reduction in cell coverage due to an increase in power update period actually translates into a decrease in voice capacity, if the $90 \%$ coverage has to be maintained. We found that the relative capacity gain of the Kalman method over the SIR method increases from $20 \%$ to $47 \%$ when the frequency of updating power is decreased from once every one packet to once every three packets. This significant improvement probably justifies the additional overhead in protocol and interference tracking of the Kalman method.

\section{Reason for Superior Performance of Kalman Method}

Let us explain why the Kalman power control (1) performs better than the SIR method (2), as shown in Figures 3 to 5. Actually, these two methods are similar. To see that, using a fact that $\beta(n-1)=p(n-1) g(n-1) / I(n-1)$ where $I(n$ $1)$ is the actual interference power for packet $n-1$, (2) becomes

$$
p(n)=\min \left\{\gamma^{*} I(n-1) / g(n-1), p_{\max }\right\} .
$$

If the path gain $g(n)$ does not change drastically from one packet to the next, (1) and (3) are similar, except that the Kalman method in (1) is based on interference prediction $\tilde{I}(n)$, while the SIR method uses the actual interference power for the last packet. In essence, the Kalman method provides some sort of smoothing on the interference measurements. If measurements contain errors, this smoothing effect can lead to performance improvements when compared with the SIR method. In case of accurate measurements and path gains unchanged in time as assumed in this study, the smoothing effect will not provide significant difference in performance.

On the other hand, for the setting considered in this paper, the major difference between the Kalman and SIR method lie on the ways they choose the transmission power for the first packet of each talk spurt. For traditional circuitswitched voice service, the selection of the first transmission power has little impacts on the overall network performance because call holding time is much longer than the power update period to ensure the "convergence" of the appropriate transmission power. However, for packet voice service with an average of 50 packets per talk spurt, the selection of the first transmission power becomes important. Since the Kalman method continuously tracks and predicts interference power, the transmission power can be appropriately selected for the first packet according to (1). In contrast, the SIR method chooses the first power to fully compensate the signal path gain, which can be quite different from the appropriate power level to combat interference. For this reason, the SIR method does not perform as well as the Kalman method does for the packet voice service. We expect that the same comment applies to other power control methods that do not base on the 
tracking and use of interference power prior to the beginning of a talk spurt.

\section{IMPLEMENTATION CONSIDERATIONS FOR EDGE SYSTEM}

\section{A. Control Messages}

The exchange of control messages for the Kalman power control has been outlined in Figures 1 and 2. Let us comment on how these messages are related to the EDGE protocols [G98]. For downlink transmission of a talk spurt, the paging message and the paging response in Figure 1 are sent on the packet paging channel $(\mathrm{PPCH})$ and the packet random access channel (PRACH), respectively. The current protocol specifications do not include the interference prediction information in the paging response message. So, the proposed Kalman method will require several bit positions (e.g., we have found that 4 to 5 bits are typically sufficient to cover a dynamic range of $30 \mathrm{~dB}$ ) for each channel under tracking in the message. The resourceassignment message and voice packets in Figure 1 are transmitted over the packet access grant channel (PAGCH) and packet data traffic channel (PDTCH), as specified in the current protocols. On the other hand, the existing specifications cannot adequately support the transmission of fast, periodic control message with updated interference prediction for the voice channel in use. One could transmit the control messages on the packet associated control channel (PACC), but its frequency is not high enough for the fast power control method. For example, Figure 4 shows that if the power update period is longer than three packets (i.e., $60 \mathrm{msec}$ ), the performance gain of power control degrades quickly. Similar to a recent proposal for measurement reporting every $60 \mathrm{msec}$ for enhanced circuitswitched data service [E99b], the Kalman power control will require such a fast and frequent transfer of updated interference prediction from the receiving MS to its BS.

For uplink transmission of voice packets, the channel request and access grant message (with the assigned channel and transmission power information) in Figure 2 can be sent via the fast packet random access channel (FPRACH) and the fast packet access grant channel (FPAGCH) proposed in [QCC00]. Similar to the downlink transmission, current protocols do not support fast and frequent transfer of updated transmission power from the $\mathrm{BS}$ to the receiving MS. One possible way is to attach the power information to the uplink state flag (USF), so that a MS knows in which time slot (by the function of USF) and at what power level it can transmit a voice packet. However, this approach represents an interim because the USF is embedded at the beginning of each downlink RLC block. Since the intended receiving MS's of the USF and the data block are likely to be different and can be located far apart, the approach of augmenting power information to the USF will not work well, for example, when smart antennas are employed to target a transmission to its intended receiver with reduced beamwidth for capacity improvement. It appears that an ideal, long-term solution would be to establish a fast control channel for frequent transfer of power-control information from BS to MS.

\section{B. Interference Power Measurements}

The Kalman power control requires both BS's and MS's to continuously measure and track interference power received from co-channel sectors. In practice, MS's can probably monitor interference power for a small number of traffic channels (e.g., a few time slots on the same frequency carrier) to conserve battery power. Without loss of generality, let us consider downlink transmission and interference measurements on the MS's. At a high level, the interference power is equal to the difference between the total received power and the power of the desired signal, where the latter can be measured by filtering based on the training sequence for the signal. It is a common practice that the same training sequence is used for transmission to any MS on a given voice channel. Since the sequence is made known to all MS's current receiving packets or tracking interference on the channel, they can apply various techniques such as [S96, p.541] and [CGM98] to measure the interference (plus noise) power.

\section{CONCLUSION AND FUTURE WORK}

In this paper, using the EDGE system as an example, we have applied the Kalman-filter power control method [L99] based on interference tracking and prediction to packet voice service. Our results reveal that power control significantly improves the spectral efficiency by enabling $1 / 3$ frequency reuse while maintaining the stringent $2 \%$ packet loss probability and $90 \%$ coverage for voice service, thus avoiding the "trunking inefficiency" of high reuse factors. More specifically, for allocated spectrum of 1.8, 3.6 and $5.4 \mathrm{MHz}$, the $1 / 3$ reuse with the Kalman power control can yield $102.5 \%, 49.5 \%$ and $32.5 \%$ improvement in spectral efficiency, respectively, over $3 / 9$ reuse (regardless of whether or not power control is used). We have also found that the Kalman method provides $20 \%$ additional spectral efficiency when compared with a SIR method and the former method is more robust than the latter for increased power update period. The protocol requirements for the implementation of the Kalman method in the EDGE system have also been discussed.

In terms of future work, despite the significant capacity gain of the Kalman power control, the current protocols and control-channel structure of the EDGE system are not adequate to provide the required fast and frequent transfer of control information. It will be desirable to enhance the protocol specifications to realize the potential performance gain. Second, we plan to apply the Kalman power control to integrated service environments, with a goal to enable 
efficient sharing of the same radio spectrum by various services such as data and voice. Since data service makes use of a link-adaptation method to improve network throughput, the challenge there is to integrate power control with link adaptation to meet the stringent performance requirements for voice service, while maximizing the overall network throughput for data service. We plan to explore and improve the algorithms of integrated power control and link adaptation proposed in [LW00a] and [LW00b] for the EDGE system. Third, as discussed in Section II, each MS can possibly track and forward the interference predictions of a few channels to its BS when requested. So, the $\mathrm{BS}$ can possibly assign the channel having the least amount of interference to the MS (e.g., [CC96]) on a per talk-spurt basis. It will be interesting to investigate the performance gain of such integrated channel assignment and power control.

\section{ACKNOWLEDGMENTS}

The authors would like to thank Li Fung Chang for providing the link error results in Table 1 and her discussion. Thanks are also due to Kapil Chawla, Larry Greenstein, Paul Henry, Nelson Sollenberger, Jim Whitehead and Jack Winters for their discussion and comments.

\section{REFERENCES}

[AC93] S. Ariyavisitakul and L. F. Chang, "Signal and Interference Statistics of a CDMA System with Feedback Power Control," IEEE Trans. on Commun., Vol. 41, Nov. 1993, pp.1626-1634.

[C99] L. F. Chang, private communications, 1999.

[CC96] M. M.-L. Cheng and J. C.-I. Chuang, "Performance Evaluation of Distributed Measurement-Based Dynamic Channel Assignment in Local Wireless Communications," IEEE J. on Select. Areas in Commun., Vol. 14, No. 4, May 1996, pp. 698-710.

[CGM98] R. L. Cupo, G. D. Golden, C. C. Martin, K. L. Sherman, N. R. Sollenberger, J. H. Winters and P. W. Wolniansky, "A Four-Element Adaptive Antenna Array for IS-136 PCS Base Stations," submitted to IEEE Trans. on Veh. Tech., 1998.

[E99a] ETSI SMG2, WPA/WPB, "EDGE: Concept Proposal for Enhanced GPRS," Tucson, AZ, May 31 to June 4, 1999, Source: Ericsson.

[E99b] ETSI SMG2, Working Session on EDGE, "Fast Measurement Reporting and Power Control for ECSD," Tdoc SMG2-2e99-331, Versailles, France, August 24 to 27, 1999, Source:Nokia.

[FHC99] T. Fingsheidt, T. Hindelang, R. V. Cox and N. Seshradi, "A Study of Voice Transmission Over EDGE Using the IS-641 Speech Coder," contribution 3G.IP/99.02.20.46, July 20, 1999, Telenor Expo, Oslo, Norway.
[FM93] G. J. Foschini and Z. Miljanic, "A Simple Distributed Autonomous Power Control Algorithm and its Convergence," IEEE Trans. on Veh. Tech., Vol. 42, No. 4, Nov. 1993, pp. 641-646.

[G98] GSM 03.64 version 6.0.0 (1998-04), Digital Cellular Telecommunications System (Phase 2+); General Packet Radio Service (GPRS); Overall Description of the GPRS Radio Interface; Stage 2.

[HWJ97] Z. J. Haas, J. H. Winters and D. S. Johnson, "Simulation Results of the Capacity of Cellular Systems," IEEE Trans. on Veh. Tech., Vol. 46, No. 4, Nov. 1997, pp. 805-817.

[L99] K. K. Leung, "A Kalman-Filter Method for Power Control in Broadband Wireless Networks," Proc. of IEEE INFOCOM'99, New York, NY, March 1999, pp. 948-956; also available at http://www.research.att.com/ kkleung/.

[LW00a] K. K. Leung and L.-C. Wang, "Controlling QoS by Integrated Power Control and Link Adaptation in Broadband Wireless Networks," European Trans. on Commun., July-Aug. 2000; also in Proc. of IEEE PIMRC'99, Osaka Japan, Sept. 1999.

[LW00b] K. K. Leung and L.-C. Wang, "Integrated Link Adaptation and Power Control for Wireless IP Network," Proc. of IEEE Veh. Tech. Conf. Spring, May 2000, Tokyo, Japan, pp. 2086-2092.

[QCC00] X. Qiu, K. Chawla, L. F. Chang, J. C.-I. Chuang, N. R. Sollenberg and J. Whitehead, "RLC/MAC Design Alternatives for Supporting Integrated Services over EGPRS," IEEE Personal Communications, April 2000, pp. 20-33.

[R96] T. S. Rappaport, Wireless Communications: Principles and Practice, New York: IEEE Press and Prentice Hall, 1996.

[RZ98] Special issue on power control, Wireless Networks 4(1998) 3, Zvi Rosberg and Jens Zander (Ed.).

[S96] G. L. Stuber, Principles of Mobile Communication, Kluwer Academic Publishers, 1996.

[SAE98] P. Schramm, H. Andreasson, C. Edholm, N. Edvardssona, M. Hook, S. Javerbring, F. Muller and J. Skold, "Radio Interference Performance of EDGE, a Proposal for Enhanced Data Rates in Existing Digital Cellular Systems," Proc. of IEEE VTC, Ottawa, Canada, May 1998, pp. 1064-1068.

[UY98] S. Ulukus and R. D. Yates, "Stochastic Power Control for Cellular Radio Systems," IEEE Trans. on Commun., Vol. 46, June 1998, pp. 784-798.

[W93] J. F. Whitehead, "Signal-level-based Dynamic Power Control for Co-channel Interference Management," Proc. of IEEE Veh. Tech. Conf., Secaucus, NJ, May 1993, pp. 499-502.

[Z92] J. Zander, "Performance of Optimum Transmitter Power Control in Cellular Radio Systems," IEEE Trans. on Veh. Tech., Vol. 41, No. 1, Feb. 1992, pp. 57-62. 